\title{
DUAL INTEGRALS IN NON-LINEAR FRACTURE MECHANICS
}

\author{
Claude Stolz \\ Laboratoire de Mécanique des Solides, CNRS UMR7649 \\ École polytechnique, Palaiseau 91128 Cedex-France
}

\begin{abstract}
We propose a dual approach in fracture mechanics based on complementary energy. The analysis of the dissipation shows that the thermodynamical force associated with the evolution of a crack is an energy release rate, form of which depends on the presence or not of mechanical discontinuities. This energy release rate is given as an integral based on free or complementary energy. We analyse the invariance of such integrals and we discuss the obtained results in elastoplasticity.
\end{abstract}

\section{INTRODUCTION}

Consider a body $\Omega$ with a crack, represented by a straight line. Around the crack tip we distinguish three domains determined by the distance from the tip.

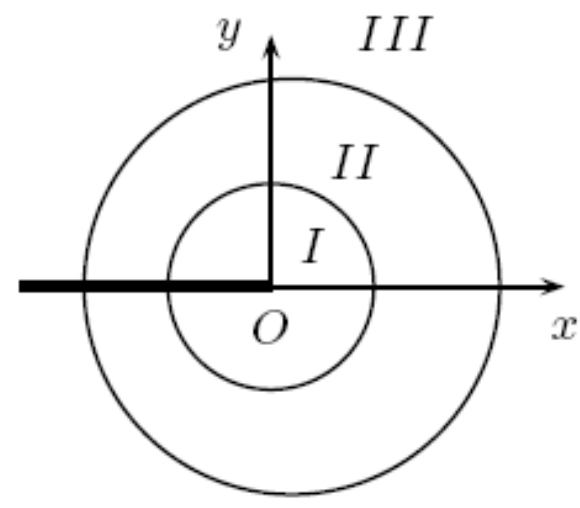

Fig. 1. The description of the behaviour near the crack tip

- The nearest zone I, where all physical processes of rupture occur, that is the process zone.

- Zone II, where the mechanical fields are described by the singular field.

- Zone III, where the mechanical fields match with boundary conditions. 
At our scale crack is represented by a line along $O x$. The normal is $\underline{e}_{y}$ in the plane, and $\underline{e}_{z}$ normal to the plane. The crack length is $l(t)$ and $a=i$ is the crack speed.

If the singularities of mechanical fields govern the crack propagation, it is not necessary to take into account the rupture process. This is an approximation which leads to a global approach of rupture. This description is powerfull and constitutes the key point for describing classical fracture mechanics. In this case, the singularities represent the loading applied on the process zone.

In non linear mechanics these notions must be revisited. The dissipation can be represented by several manner, depending on the local constitutive law and on the mechanisms of rupture. The dissipation can be concentrated in one point due to singularities or along a surface of discontinuities as in shock waves or formed inside a volume. Any situation depends on the characteristic of the material and on condition of propagation.

For example, under anti-plane shear in elastodynamics, the displacement $\underline{u}=w(x, y, t) \underline{e}_{z}$ is governed by

$$
\left(1-\frac{a^{2}}{C^{2}}\right) w_{, x x}^{\prime \prime}+w_{, y y}^{\prime \prime}=0
$$

where the Mach number $M=\frac{a}{C}$ defines the nature of this equation. For small $M<1$ the equation is elliptic, $w$ is singular and the dissipation is concentrated at the crack tip. For large value of $\mathrm{M}(M>1)$ the equation is hyperbolic, the gradient $\nabla w$ has jump and the dissipation is concentrated along moving surface.

In elastoplasticity, the local equations are also hyperbolic, the same can be realized for hyperelastic material [1].

\section{CHARACTERIZATION OF THE PROPAGATION}

The essential difficulty of the problem of propagation is the dependence of $\Omega$ on the crack length and the presence of moving singularities accompanying the crack.

One possibility has been investigated in [2] by introducing a geometrical lagrangean description. We propose to based our description upon the concept of singularity transport $([3],[4],[5])$. Inside a moving frame in translation with the crack tip, the nature of the singularity is conserved. The crack singularity is surrounded by a curve $\Gamma$ delimiting a domain $V_{\Gamma}$. This domain translates with the tip of the crack position of which is given by function $l(t)$. All mechanical quantities are expressed in terms of the classical fixed coordinates outside $V_{\Gamma}$ and in terms of moving coordinates inside $V_{\Gamma}$.

$$
\begin{aligned}
& x=X-l(t), \\
& y=Y .
\end{aligned}
$$

Any mechanical quantity $F$ possesses time derivative given by $\stackrel{\circ}{f}$, which represents the variation of $f$ in the moving frame :

$$
F(X, Y, t)=f(x, y, t), \quad \dot{f}=\frac{\partial F}{\partial t}=-a \frac{\partial f}{\partial X}+\stackrel{\circ}{f} .
$$




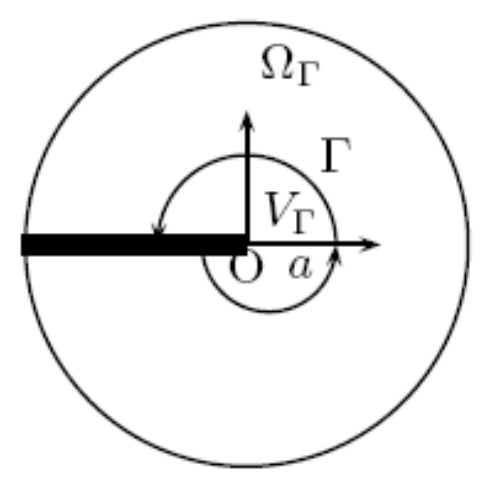

Fig. 2. Decomposition of $\Omega$ in $\Omega_{\Gamma} \cup V_{\Gamma}$

In order to separate the contribution of the crack tip in the expression of dissipation, we apply these definitions to average quantities on the whole domain:

$$
\begin{aligned}
\mathcal{F} & =\int_{\Omega} f \rho \mathrm{d} \Omega=\int_{\Omega_{\Gamma}} f \rho \mathrm{d} \Omega+\int_{V_{\Gamma}} f \rho \mathrm{d} \Omega, \\
\frac{\mathrm{d}}{\mathrm{dt}} \mathcal{F} & =\frac{\mathrm{d}}{\mathrm{dt}}\left(\int_{\Omega_{\Gamma}} f \rho \mathrm{d} \Omega\right)+\int_{V_{\Gamma}} \stackrel{\circ}{f} \rho \mathrm{d} \Omega . \\
\frac{\mathrm{d}}{\mathrm{dt}}\left(\int_{\Omega_{\Gamma}} f \rho \mathrm{d} \Omega\right) & =\int_{\Omega_{\Gamma}} \dot{f} \rho \mathrm{d} \Omega-\int_{\Gamma} f \rho a n_{x} \mathrm{~d} S .
\end{aligned}
$$

Let us introduce the notations : $f_{x}=f \cdot \underline{e}_{x}, \nabla_{x} f=\nabla f \cdot \underline{e}_{x}$.

Dissipation. The dissipation of the whole system can be rewritten as

$$
D_{m}=\mathcal{P}_{e}-\frac{\mathrm{d}}{\mathrm{dt}} \int_{\Omega} w \rho \mathrm{d} \Omega \geq 0,
$$

where the power of external forces $\mathcal{P}_{e}$ is given in term of local stresses, taking into account the conservation of the momentum :

$$
\mathcal{P}_{e}=\int_{\partial \Omega} \underline{n} \cdot \sigma \cdot \underline{v} \mathrm{~d} S
$$

Using divergence theorem, this quantity is decomposed in two terms:

$$
\int_{\partial \Omega} \underline{n} \cdot \sigma \cdot \underline{v} \mathrm{~d} S=\int_{\Omega_{\Gamma}} \sigma: \varepsilon(\underline{v}) \mathrm{d} \Omega+\int_{\Gamma} \underline{n} \cdot \sigma \cdot \underline{v} \mathrm{~d} S .
$$

Using now the decomposition of the volume $\left(\Omega=\Omega_{\Gamma} \cup V_{\Gamma}\right)$ we obtain first

$$
\frac{\mathrm{d}}{\mathrm{dt}} \int_{\Omega} w \rho \mathrm{d} \Omega=\frac{\mathrm{d}}{\mathrm{dt}}\left(\int_{\Omega_{\Gamma}} w \rho \mathrm{d} \Omega\right)+\int_{V_{\Gamma}} \stackrel{\circ}{w} \rho \mathrm{d} \Omega
$$


and by application of general relations :

$$
\begin{aligned}
\frac{\mathrm{d}}{\mathrm{dt}} \int_{\Omega_{\Gamma}} w \rho \mathrm{d} \Omega & =\int_{\Omega_{\Gamma}} \dot{w} \rho \mathrm{d} \Omega-\int_{\Gamma} \rho w n_{x} \mathrm{~d} S a, \\
\int_{V_{\Gamma}} \stackrel{\circ}{w} \rho \mathrm{d} \Omega & =\int_{\Gamma} \underline{n} \cdot \sigma \cdot \underline{\circ} \mathrm{d} S+o(R),
\end{aligned}
$$

where we have taken account of the traction-free boundary condition $\sigma \cdot \underline{n}=0$ along the crack lips. The quantity $o(R)$ is regular and tends to zero with the radius of the volume $V_{\Gamma}$.

The dissipation is rewritten finally as

$$
\begin{aligned}
D_{m} & =\int_{\Omega_{\Gamma}}(\sigma: \varepsilon(\underline{v})-\rho \dot{w}) \mathrm{d} \Omega \\
& +\int_{\Gamma}\left(\underline{n} \cdot \sigma \cdot(\underline{v}-\underline{\circ})+\rho w \underline{n} \cdot \underline{e}_{x} a\right) \mathrm{d} S+o(R) \geq 0 .
\end{aligned}
$$

The displacement $\underline{u}$ is continuous along the curve $\Gamma$. The condition of compatibility implies Hadamard relations on the rates :

$$
[\underline{u}]=0 \Rightarrow \underline{v}=\underline{\circ}-\nabla_{x} \underline{u} a .
$$

Then the dissipation is decomposed in two terms : a volume part due to irreversibility over the body and a surface term associated with the propagation of the crack :

$$
\begin{aligned}
D_{m} & =\lim _{R \rightarrow 0} \int_{\Omega_{\Gamma}}(\sigma: \varepsilon(\underline{v})-\rho \dot{w}) \mathrm{d} \Omega \\
& +\lim _{R \rightarrow 0} \int_{\Gamma}\left(-\underline{n} \cdot \sigma \cdot \nabla_{x} \underline{u}+\rho w n_{x}\right) \mathrm{d} S \quad a \geq 0 .
\end{aligned}
$$

Case of linear elasticity. In this case the local behaviour is reversible and the stress $\sigma=$ $\rho \frac{\partial w}{\partial \varepsilon}$, then there is no dissipation in the volume :

$$
\int_{\Omega_{\Gamma}}(\sigma: \varepsilon(\underline{v})-\rho \dot{w}) \mathrm{d} \Omega=0 .
$$

When $\Gamma$ is reduced to the crack tip, the result is conserved :

$$
\lim _{R \rightarrow 0} \int_{\Omega_{\Gamma}}(\sigma: \varepsilon(\underline{v})-\rho \dot{w}) \mathrm{d} \Omega=0 .
$$

The global dissipation contains only the contribution of the crack :

$$
D_{m}=\lim _{R \rightarrow 0} \int_{\Gamma}\left(-\underline{n} \cdot \sigma \cdot \nabla_{x} \underline{u}+\rho w n_{x}\right) \mathrm{d} S a .
$$

The thermodynamical force associated with the propagation is the free energy release rate $\mathcal{G}$ defined by :

$$
\mathcal{G}=\lim _{R \rightarrow 0} \int_{\Gamma}\left(-\underline{n} \cdot \sigma \cdot \nabla_{x} \underline{u}+\rho w n_{x}\right) \mathrm{d} S .
$$


Invariance of J-integral. Consider now $S$ a closed loop inside a domain $\Omega$, over which the body forces are null. For an homogeneous linearly elastic material, the density $\rho$ is uniform. The stresses satisfy both the equations of state and the conservation of the momentum :

$$
\sigma=\rho \frac{\partial w}{\partial \varepsilon}, \quad \operatorname{div} \sigma=0 \text { over } \Omega
$$

Consider the integral $C$

$$
C=\int_{S}\left(\rho w n_{k}-\sigma_{i j} u_{i, k} n_{j}\right) \mathrm{d} S
$$

then by divergence theorem the integral is equal to

$$
\int_{\Omega_{S}} \frac{\partial(\rho w)}{\partial x_{k}}-\frac{\partial\left(\sigma_{i j} u_{i, k}\right)}{\partial x_{j}} \mathrm{~d} \Omega=\int_{\Omega_{S}}\left(\sigma_{i j} \varepsilon_{i j, k}-\sigma_{i j} u_{i, j k}-\frac{\partial \sigma_{i j}}{\partial x_{j}} u_{i, k}\right) \mathrm{d} \Omega .
$$

Using now the conservation of momentum, we find that the integral $C$ is null.

From this result, it follows that the integral $J_{\Gamma}$ :

$$
J_{\Gamma}=\int_{\Gamma}\left(\rho w n_{k}-\sigma_{i j} u_{i, k} n_{j}\right) \mathrm{d} S,
$$

is independent of the choice of the loop $\Gamma$, taking account of the free-traction conditions along the lips of the crack. Therefore

$$
J_{\Gamma}=\lim _{\Gamma \rightarrow 0} J_{\Gamma}=\mathcal{G} .
$$

Examples of non linear behaviour. For some classes of non linear material, the gradient of the displacement presents discontinuities along surface $S$. In this case, the limit $R \rightarrow 0$ must take these discontinuities into account. The jumps of gradient of displacement imply jumps on velocities

$$
[\underline{v}]_{S}+a\left[\underline{u}_{1}\right]_{S}=0
$$

In perfect plasticity [[3]] the release rate of energy determined by the dissipation is given by

$$
J_{s}=\lim _{\Gamma \rightarrow S} \int_{\Gamma}\left(w\left(\varepsilon-\varepsilon_{p}\right) n_{1}-\underline{n} \cdot \sigma \cdot \underline{u}_{, 1}\right) \mathrm{d} s
$$

where $S$ is the surface along which the gradient of displacement has discontinuities:

$$
[\underline{\dot{u}}]_{S}+a\left[\underline{u}_{, 1}\right]_{S}=0
$$

$S$ is a shock surface or a shock line.

\section{ENERGETICAL INTERPRETATION}

For non linear material, the strain energy $w$ depends on the strain $\varepsilon$ and on internal parameters $\alpha$ which describe hardening behaviour. The potential energy for the system is :

$$
\mathcal{E}\left(\underline{u}, \alpha, l, \underline{\mathrm{T}}^{d}\right)=\int_{\Omega} \rho w(\varepsilon(\underline{u}), \alpha) \mathrm{d} \Omega-\int_{\partial \Omega_{\mathrm{T}}} \underline{\mathrm{T}}^{d} \cdot \underline{u} \mathrm{~d} S,
$$

and the dissipation is rewritten as

$$
D_{m}=\mathcal{P}_{e}-\frac{\mathrm{d}}{\mathrm{dt}}\left(\mathcal{E}+\int_{\partial \Omega_{\mathrm{T}}} \underline{\mathrm{T}}^{d} \cdot \underline{u} \mathrm{~d} S\right) \geq 0
$$


Taking account of the derivation of the potential energy with respect to its arguments, we obtain :

$$
\frac{\mathrm{d}}{\mathrm{dt}}(\mathcal{E})=\frac{\partial \mathcal{E}}{\partial \underline{u}} \cdot \underline{v}+\frac{\partial \mathcal{E}}{\partial \alpha} \cdot \dot{\alpha}+\frac{\partial \mathcal{E}}{\partial l} a+\frac{\partial \mathcal{E}}{\partial \underline{\mathrm{T}}^{d}} \cdot \underline{\dot{\mathrm{T}}}^{d}
$$

As

$$
\begin{aligned}
& \frac{\partial \mathcal{E}}{\partial \underline{u}} \cdot \underline{v}=\int_{\partial \Omega} \underline{n} \cdot \sigma \cdot \underline{v}-\int_{\partial \Omega_{\mathrm{T}}} \underline{\mathrm{T}}^{d} \cdot \underline{v} \mathrm{~d} S \\
& \frac{\partial \mathcal{E}}{\partial \alpha} \cdot \dot{\alpha}=\int_{\Omega} \rho \frac{\partial w}{\partial \alpha} \dot{\alpha} \mathrm{d} \Omega
\end{aligned}
$$

then

$$
D_{m}=-\frac{\partial \mathcal{E}}{\partial \alpha} \cdot \dot{\alpha}-\frac{\partial \mathcal{E}}{\partial l} a \geq 0 .
$$

Release rate of energy. For the system, the crack length plays the role of an internal parameter. The thermodynamical force associated with the propagation is the release rate of energy $\mathcal{G}$ obtained by the global state equation :

$$
\mathcal{G}=-\frac{\partial \mathcal{E}}{\partial l}
$$

In dynamical evolution the potential energy of the wall system must be replaced by the Hamiltonian [4].

\section{DUAL APPROACH IN LINEAR ELASTICITY}

We study the propagation of a straight crack in direction $\underline{e}_{1}$ in a continuum $\Omega$. On the boundary $\partial \Omega_{\mathrm{T}}$, the stress $\underline{T}^{d}$ is imposed and on the complementary part $\partial \Omega_{\mathrm{u}}$ the displacement is prescribed. The crack is stress free. In classical brittle fracture, the constitutive behaviour is linear elastic. The strain energy $w(\varepsilon)$ is a quadratic function of the strain $\varepsilon(\underline{u})$ which is associated to the displacement $\underline{u}$ :

$$
w(\varepsilon)=\frac{1}{2} \varepsilon . \mathbf{C} \cdot \varepsilon, \quad \varepsilon=\frac{1}{2}\left(\operatorname{grad} \underline{u}+\operatorname{grad}^{t} \underline{u}\right) .
$$

We denote by $\sigma$ the value of the stress tensor associated with the strain $\sigma=\mathbf{C}: \varepsilon$.

The evolution law for the crack is given in terms of energy release rate $\mathcal{G}$ and satisfies the normality rule $\mathcal{G}-G_{c} \leq 0 ; a \geq 0 a\left(\mathcal{G}-G_{c}\right)=0$; where $G_{c}$ is a characteristic of the material

The energy release rate $\mathcal{G}$ is given in terms of potential energy $\mathcal{E}$

$$
\begin{aligned}
\mathcal{E}(\underline{u}, l) & =\int_{\Omega(l)} w(\varepsilon) \mathrm{d} \Omega-\int_{\partial \Omega_{\mathrm{T}}} \underline{\mathrm{T}}^{d} \cdot \underline{u} \mathrm{~d} s, \\
\mathcal{G} & =-\frac{\partial \mathcal{E}}{\partial l}=\int_{\Gamma}\left(w(\varepsilon) n_{1}-\underline{n} \cdot \sigma \cdot u_{, 1}\right) \mathrm{d} s=J_{\Gamma}
\end{aligned}
$$

A state of equilibrium for a given lenght of crack $l$ is defined by a displacement which minimises $\mathcal{E}$ on the set of displacements $\underline{u}$ such that $\underline{u}=\underline{u}^{d}$ along $\partial \Omega_{\mathrm{u}}$. The equilibrium solution is $\underline{u}^{s o l}\left(l, \underline{u}^{d}, \underline{T}^{d}\right)$. 
In the dual approach, the complementary energy is defined in terms of stresses

$$
\mathcal{E}^{*}(\sigma, l)=-\int_{\Omega(l)} w^{*}(\sigma) \mathrm{d} \Omega+\int_{\partial \Omega_{\mathrm{u}}} \underline{n} \cdot \sigma \cdot \underline{u}^{d} \mathrm{~d} s .
$$

Then the release rate of energy is the Bui's dual integral [6]:

$$
I_{\Gamma}=J_{\Gamma}=\int_{\Gamma}\left(-w^{*}(\sigma) n_{1}+\underline{n} \cdot \sigma_{, 1} \cdot \underline{u}\right) \mathrm{d} s .
$$

The equality of the two integral is due to the fact that at a state of equilibrium for the same crack length and the same boundary conditions, the potential energy and the complementary energy are equals. This results is obtained taking account of the classical relations

$$
w^{*}(\sigma)+w(\varepsilon)=\sigma \cdot \varepsilon, \quad \int_{V_{c}} \sigma: \varepsilon(\underline{u}) \mathrm{d} \Omega=\int_{\mathcal{C}} \underline{n} \cdot \sigma \cdot \underline{u} \mathrm{~d} s .
$$

Lemma : For all stresses $\tilde{\sigma}$ which satisfy the equilibrium equation $(\operatorname{div} \tilde{\sigma}=0)$, and for all strains $\hat{\varepsilon}$ such that $\left(2 \hat{\varepsilon}=\operatorname{grad} \underline{\hat{u}}+\operatorname{grad}^{t} \underline{\hat{\hat{u}}}\right)$ and for all closed volume with boundary $C$, we have:

$$
\int_{\mathcal{C}}\left(\tilde{\sigma}: \hat{\varepsilon} n_{i}-\underline{n} \cdot \tilde{\sigma}_{, i} \cdot \underline{\hat{u}}-\underline{n} \cdot \tilde{\sigma} \cdot \underline{\hat{u}}_{, i}\right) \mathrm{d} s=0
$$

The two integrals $I_{\Gamma}$ et $J_{\Gamma}$ are independent of the close curve $\Gamma$. This can be shown considering a close curve $C$ composed by two curves $\Gamma_{1}$ and $\Gamma_{2}$ surrounding the crack tip $\left(V_{\Gamma_{1}} \subset V_{\Gamma_{2}}\right)$ and by the crack lips.

We can introduce the local Eshelby tensors $p$ and $p^{*}$ :

$$
p=w \mathbf{I}-\sigma \cdot \nabla \underline{u}, \quad p^{*}=-w^{*} \mathbf{I}+\nabla \sigma \cdot \underline{u}
$$

They satisfy

$$
0=\int_{V_{c}} \operatorname{div} p \mathrm{~d} \Omega=\int_{\mathcal{C}} \underline{n} \cdot p \mathrm{~d} s, \quad 0=\int_{V_{c}} \operatorname{div} p^{*} \mathrm{~d} \Omega=\int_{V_{c}} \underline{n} \cdot p^{*} \mathrm{~d} s
$$

For the equilibrium solution $w(\varepsilon)=w^{*}(\sigma)=\frac{1}{2} \sigma: \varepsilon$ and then we obtain

$$
p-p^{*}=\sigma: \varepsilon \mathbf{I}-(\sigma \cdot \nabla \underline{u}+\nabla \sigma \cdot \underline{u}) ; \quad p+p^{*}=\nabla \sigma \cdot \underline{u}-\sigma . \nabla \underline{u}
$$

On the crack lips the contributions $\underline{n} \cdot p \cdot \underline{e}_{1}$ and $\underline{n} \cdot p^{*} \cdot \underline{e}_{1}$ vanish. This induces the invariance of integrals $I_{\Gamma}$ and $J_{\Gamma}$ with respect to the choice of $\Gamma$, the constant values of which are denoted by $I$ and $J$ respectively. Taking the properties of $p$ and $p^{*}$ and the lemma into account, for an equilibrium solution we obtain:

$$
\begin{aligned}
I+J & =\int_{\Gamma}\left(\underline{n} \cdot \sigma, 1 \cdot \underline{u}-\underline{n} \cdot \sigma \cdot \underline{u}_{, 1}\right) \mathrm{d} s \\
J-I & =\int_{\Gamma}\left(\left(w+w^{*}\right) n_{1}-\underline{n} \cdot \sigma, 1 \cdot \underline{u}-\underline{n} \cdot \sigma \cdot \underline{u}_{, 1}\right) \mathrm{d} s=0
\end{aligned}
$$




\section{DUAL APPROACH IN ELASTOPLASTICITY}

Case of perfect plasticity. The local behaviour is given by the free energy $w\left(\varepsilon-\varepsilon_{p}\right)$ where $\varepsilon_{p}$ is the plastic strain, which evolution is governed by a normality rule associated with a convex function $f(\sigma) \leq 0$ such that

$$
f(\sigma) \leq 0 ; \lambda>0, \dot{\varepsilon}_{p}=\lambda \frac{\partial f}{\partial \sigma}, \quad \lambda f=0 .
$$

We introduce the complementary energy for perfect plasticity:

$$
w^{*}(\sigma)=\sigma:\left(\varepsilon-\varepsilon^{p}\right)-w\left(\varepsilon-\varepsilon^{p}\right)
$$

The definitions of both global energies $\mathcal{E}$ and $\mathcal{E}^{*}$ are conserved with these new local densities. For a given field of internal parameter, the equilibrium solution minimizes the potential or the complementary energy, then it is obvious that

$$
I_{s}=J_{s}=-\frac{\partial \mathcal{E}}{\partial l}=-\frac{\partial \mathcal{E}^{*}}{\partial l}
$$

where the thermodynamical force $J_{s}$ is given in eq 22 . Introducing the Eshelby tensors $p$ and $p^{*}$, they satisfy now

$$
0=\int_{V_{c}} \operatorname{div} p \mathrm{~d} \omega+\int_{V_{c}} \sigma: \nabla \varepsilon^{p} \mathrm{~d} \omega \quad 0=\int_{V_{c}} \operatorname{div} p^{*} \mathrm{~d} \omega-\int_{V_{c}} \nabla \sigma: \varepsilon^{p} \mathrm{~d} \omega
$$

for any closed volume which does not contain discontinuities. Using the properties of $p$ and $p^{*}$ the two integrals

$$
\mathcal{G}=J_{\Gamma}+\int_{\Omega_{\Gamma}} \sigma: \varepsilon_{, 1}^{p} \mathrm{~d} \Omega, \quad \mathcal{G}^{*}=I_{\Gamma}-\int_{\Omega_{\Gamma}} \sigma_{, 1}: \varepsilon^{p} \mathrm{~d} \Omega
$$

do not depend on $\Gamma$, and we find:

$$
\begin{aligned}
& \mathcal{G}+\mathcal{G}^{*}=2 J_{s}=\int_{\Gamma}\left(\underline{n} \cdot \sigma_{, 1} \cdot \underline{u}-\underline{n} \cdot \sigma \cdot \underline{u}_{, 1}\right) \mathrm{d} S+\int_{\Omega_{\Gamma}}\left(\sigma: \varepsilon_{, 1}^{p}-\sigma_{, 1}: \varepsilon^{p}\right) \mathrm{d} \Omega \\
& \mathcal{G}-\mathcal{G}^{*}=0=\int_{\Gamma}\left(\left(w+w^{*}\right) n_{1}-\underline{n} \cdot \sigma, 1 \cdot \underline{u}-\underline{n} \cdot \sigma \cdot \underline{u}_{1}\right) \mathrm{d} S+\int_{\Omega_{\Gamma}}\left(\sigma: \varepsilon^{p}\right)_{, 1} \mathrm{~d} \Omega .
\end{aligned}
$$

Adding these equations, we then take the limit $\Gamma \rightarrow S$ to obtain

$$
\mathcal{G}+\mathcal{G}^{*}=I_{s}+J_{s}=\int_{S}\left(\underline{n} \cdot\left[\sigma_{, 1}\right]_{S} \cdot \underline{u}-\underline{n} \cdot \sigma \cdot\left[\underline{u}_{1}\right]_{S}\right) \mathrm{d} s ;
$$

therefore we have

$$
I_{s}=\lim _{\Gamma \rightarrow S} \int_{\Gamma}\left(-w^{*}(\sigma) n_{1}+\underline{n} \cdot \sigma_{, 1} \cdot \underline{u}\right) \mathrm{d} s .
$$

This generalizes the Bui's dual-integral to perfect plasticity. 
Elastoplasticity with hardening. The local behaviour is defined by the local free energy $w(\varepsilon, \alpha)$ where $\alpha$ is a set of internal parameters, and $w$ is assumed to be convex of it's argument. The thermodynamical forces associated with these state variables are the stress $\sigma$ and the internal force $A$

$$
\sigma=\frac{\partial w}{\partial \varepsilon}, \quad A=-\frac{\partial w}{\partial \alpha}
$$

The complementary energy is defined as previously by

$$
w^{*}(\sigma, A)=\sigma: \varepsilon-A . \alpha-w(\varepsilon, \alpha) .
$$

For the equilibrium solution the stress $\sigma$ satisfy the equilibrium $\operatorname{div} \sigma=0$ and the boundary conditions $\underline{n} . \sigma=\underline{\mathrm{T}}^{d}$. The internal forces $A$ are inside the domain of reversibility.

The Eshelby-tensors $p$ and $p^{*}$ satisfy the properties

$$
0=\int_{V_{c}} \operatorname{div} p \mathrm{~d} \Omega+\int_{V_{c}} A . \nabla \alpha \mathrm{d} \Omega ; \quad 0=\int_{V_{c}} \operatorname{div} p^{*} \mathrm{~d} \Omega-\int_{V_{c}} \nabla A . \alpha \mathrm{d} \Omega
$$

and by a reasoning analogous to those of perfect plasticity

$$
\mathcal{G}=J_{\Gamma}+\int_{\Omega_{\Gamma}} A \cdot \alpha_{, 1} \mathrm{~d} \Omega, \quad \mathcal{G}^{*}=I_{\Gamma}-\int_{\Omega_{\Gamma}} A_{, 1}: \alpha \mathrm{d} \Omega .
$$

The property $\mathcal{G}=J_{s}$ is conserved.

When the energy $w$ is a quadratic function, at the equilibrium solution we have $\left(w=w^{*}=\frac{1}{2} \sigma: \varepsilon-\frac{1}{2} A: \alpha\right)$ and we obtain the peculiar form

$$
\mathcal{G}=\frac{1}{2} \int_{\Gamma}\left(\underline{n} \cdot \sigma_{, 1} \cdot \underline{u}-\underline{n} \cdot \sigma \cdot \underline{u}_{1}\right) \mathrm{d} S+\frac{1}{2} \int_{\Omega_{\Gamma}}\left(A: \alpha_{, 1}-A_{, 1}: \alpha\right) \mathrm{d} \Omega .
$$

\section{APPLICATION TO THE EVOLUTION OF CRACKS}

For describing the evolution of cracks it is necessary to have an evolution law. We consider that the evolution of a crack is governed by a generalized Griffith law

$$
J_{s} \leq G_{c}, a=0, \quad J_{s}=G_{c}, a \geq 0 .
$$

The evolution is governed by a normality rule : when $J_{s}=G_{c}$ then

$$
\dot{J}_{s}(a-\mu) \geq 0, \forall \mu \geq 0,
$$

We need to charaterize the rate $\dot{J}_{s}$.

We consider the volume $V_{\Gamma}$ translating with the velocity $a$. The rate $f$ is the rate of $f$ in the moving frame $V_{\Gamma}$. The surface $S$ has the same velocity of translation, then it's normal velocity is $a \underline{e}_{1} . \underline{n}$. In the moving frame the variation of $J_{s}$ is well defined:

$$
\dot{\mathcal{G}}=\int_{S}[\sigma]_{S}: \nabla \underline{\circ}-\stackrel{\circ}{\sigma}:[\nabla \underline{u}]_{S}-[A \stackrel{\circ}{\alpha}]_{S} \mathrm{~d} s,
$$

which can be rewritten as

$$
\dot{\mathcal{G}}=\int_{\Gamma}\left(\underline{n} \cdot \sigma_{, 1} \cdot \underline{\circ}-\underline{\underline{u}}-\stackrel{\circ}{\sigma} \cdot \underline{u}_{, 1}\right) \mathrm{d} s+\int_{\Omega_{\Gamma}}\left(\stackrel{\circ}{A}: \alpha_{, 1}-A_{, 1}: \stackrel{\circ}{\alpha}\right) \mathrm{d} \Omega .
$$


The last term contains only coupling terms between strain and hardening, as we can shown

$$
-\stackrel{\circ}{A}: \alpha_{, 1}+A_{, 1}: \stackrel{\circ}{\alpha}=\frac{\partial^{2} w}{\partial \alpha \partial \varepsilon} \bullet\left(\stackrel{\circ}{\varepsilon} \otimes \alpha_{, 1}-\stackrel{\circ}{\alpha} \otimes \varepsilon_{, 1}\right)=\stackrel{\circ}{\sigma}: \varepsilon_{, 1}-\sigma_{, 1}: \stackrel{\circ}{\varepsilon},
$$

then the rate is given by

$$
\dot{\mathcal{G}}=\int_{\Gamma}\left(\underline{n} \cdot \sigma, 1 \cdot \stackrel{\circ}{\underline{u}}-\underline{n} \cdot \stackrel{\circ}{\sigma} \cdot \underline{u}_{, 1}\right) \mathrm{d} s+\int_{\Omega_{\Gamma}}\left(\stackrel{\circ}{\sigma}: \varepsilon_{, 1}-\sigma_{, 1}: \stackrel{\circ}{\varepsilon}\right) \mathrm{d} \Omega .
$$

This generalized the previous case obtained in [3] for perfect plasticity.

\section{CONCLUSION}

We have generalized the Bui's dual integral to elastoplastic materials. We have connected this integral to the release rate of potential and complementary energy in case of elastoplastic behaviour with hardening.

With these definitions, the formulation of the evolution of cracks are determined by a variational inequality as shown in [7]. The methods are identical as proposed in ([8],[9]) and allows stability and bifurcation analysis of a system of cracks.

\section{REFERENCES}

[1] James K. Knowles, The finite antiplane shear field near the tip of a crack for a class of incompressible elastic solids, Int. Journ. of Fracture, 13 (1977):611-639.

[2] Philippe Destuynder, M. Djaoua, Sur une interprétation mathématique de l'intégrale de Rice en théorie de la rupture fragile, Math. Appl. Sci., 3 (1981):70-77.

[3] Nguyen Quoc Son, (1985), Critère de propagation en rupture ductile, Comptes Rendus Académie des Sciences Paris, 301 (1985):567-570,

[4] Stolz Claude, Rachel-Marie Pradeilles-Duval, Approche énergétique de la propagation dynamique de discontinuités mécaniques, Comptes Rendus Académie des Sciences Paris, Série IIb, 322 (1996):525-532.

[5] Stolz Claude, Energy Methods in Non Linear Mechanics, Lecture Notes 11, Centre of Excellence for Advanced Materials ans Structures, IPPT, Polish Academy of Sciences, 2004.

[6] Bui Huy Duong, Dualité entre les intégrales indépendantes du contour dans la théorie ds solides fissurés, Comptes Rendus Académie des Sciences Paris, Série A, 376(1973):1425-1428.

[7] Stolz Claude, Sur le problème de propagation de fissure en élastoplasticité : approches primale et duale, C.R. Mécanique, 336 (2008):500-505.

[8] Nguyen Quoc Son, Stolz Claude, Sur le problème d'évolution en vitesse de propagation de fissure et de déplacement en rupture fragile ou ductile, Comptes Rendus Académie des Sciences Paris, 301 (1985):661-664.

[9] Nguyen Quoc Son, Stolz Claude, Debruyne Gilles, Energy methods in fracture mechanics : stability, bifurcation and second variations, Eur. J. Mech, A/Solids 9 (1990) 2:157-173.

[10] Lubarda V.A., Markenscoff Xanthippi, Dual conservation integrals and energy release rates, Int. Journal of Solids and Structures, 44 (2007):4079-4091.

[11] Stolz Claude, Intégrale duale en mécanique de la rupture, C.R. Mécanique, 336 (2008):434439. 


\section{TÍCH PHÂN ĐỐI NGẪU TRONG CƠ HỌC PHÁ HƯY PHI TUYẾN}

Chúng tôi đề xuất một hướng tiếp cận đối ngẫu trong cơ học phá hủy dựa trên năng lượng phụ. Sự phân tích tiêu tán năng lượng chỉ ra rằng lực nhiệt động kết hợp với sự phát triển của vết nứt là tốc độ giải phóng năng lượng tự do, mà dạng của nó phụ thuộc vào sự có mặt hoặc không có mặt của sự không liên tục về mặt cơ học. Tốc độ giải phóng năng lượng tự do này được cho dưới dạng tích phân dựa trên năng lượng tự do hoặc năng lượng phụ. Chúng tôi phân tích tính bất biến của những tích phân này và chúng tôi thảo luận các kết quả thu được trong bài toán đàn dẻo. 